\title{
The impact of process re-engineering on patient throughput in emergency departments in the UK
}

\author{
Ashis Banerjee • David Mbamalu • Geoff Hinchley
}

Received: 7 May 2008 / Accepted: 21 July 2008 / Published online: 24 September 2008

(C) Springer-Verlag London Ltd 2008

\begin{abstract}
Background The re-engineering of emergency department (ED) processes in the UK since 2002 has produced significant reductions in waiting times.

Aims We aim to describe the generic themes contributory to this improvement in performance, which has led to progress not yet replicated elsewhere in the English-speaking world. Methods We reviewed the Emergency Services Collaborative (ESC) set up by the National Health Service (NHS) Modernisation Agency as well as our own departmental performance in order to identify key themes for discussion. In addition, we reviewed relevant information from the UK Department of Health website. We used the 4-h target of patient passage through the ED as our primary outcome measure.

Results Early results from the ESC showed improvements, which have been sustained and enhanced since inception. We use our hospital performance figures to demonstrate a pattern of progressive improvement in performance, with $99.1 \%$ of all new attenders in 2007-2008 being seen, treated and discharged or admitted within $4 \mathrm{~h}$ of presentation to the ED.

Conclusions The whole systems approach to re-engineering emergency care has led to universal improvements in patient throughput in EDs in the UK. Several of the concepts found to be useful in the NHS are worthy of
\end{abstract}

\footnotetext{
A. Banerjee $(\bowtie) \cdot$ D. Mbamalu $\cdot$ G. Hinchley

Department of Emergency Medicine, Chase Farm Hospital,

The Ridgeway,

Enfield,

EN2 8JL North London, UK

e-mail: libra19542003@yahoo.co.uk
}

consideration and adoption by other health care systems. Long waits in the ED are a thing of the past in the UK.

Keywords UK · 4-h targets · Breaches · Escalation ·

Process mapping

\section{Introduction}

The 1980s and 1990s saw a progressive increase in emergency department (ED) attendances in the UK, leading to increased work load and departmental crowding. The resultant media backlash highlighted on an almost daily basis the plight of patients, often elderly, who spent long periods on trolleys in ED corridors. Service modernisation, with improved responsiveness to patient needs, was felt to be essential.

The linkage of quality of care to increased throughput, achieved through process re-engineering, is one of the success stories of the National Health Service (NHS) of the UK. While these improvements are the result of a whole systems approach, we will focus primarily on the role of the $\mathrm{ED}$ in the process.

\section{Methods}

The Emergency Services Collaborative (ESC) Programme [1] was set up as a national programme by the NHS Modernisation Agency. The ESC was to work with every acute NHS Trust to ensure that by the end of $200498 \%$ of patients were seen, treated and discharged, transferred or admitted within $4 \mathrm{~h}$ of arrival in the ED. All NHS acute hospitals were allocated the resources to test and implement changes to meet this NHS Plan target. 
Improvements were derived from both individual organisations and departments, and from national programmes of the Modernisation Agency, such as the IDEA (Ideal Design of Emergency Access) programme [2].

The programme was rolled out progressively in six waves, with 30-35 acute hospital sites per wave. The first wave began in October 2002, with all waves fully operational by July 2003 . The process allowed front-line staff to engage with managers, allowing implementation of recommended changes. Process mapping, with analysis of delays and constraints within the system, guided the re-engineering of patient assessment and management pathways.

Four key patient flows through emergency care were identified by expert consensus, which will be used for the purposes of discussion:

1. Patients with a minor injury or illness, who could be treated and discharged relatively quickly, often following a simple diagnostic assessment

2. Patients who require a longer assessment and observation in addition to diagnosis and treatment

3. Medical patients who required admission to acute hospital, with a significant length of stay

4. Patients admitted for an emergency surgical procedure

Some key themes were identified to assist in optimising patient flows (see Box 1).

Box 1

Key themes cutting across the groups:

- Streaming of care to the most appropriate provider, determined by rapid assessment at first point of contact by a nurse who replaced the more formal roles of the triage nurse

- See and treat

- Early access to diagnostics, with prioritisation of ED requests

- Improved senior and middle grade staffing of EDs

- Blurring of the boundaries between health care professionals in emergency care

- Escalation policies

- Proactive discharge planning

- Whole systems multi-disciplinary input

- Breach analysis on a daily basis

Early data from the Emergency Services Collaborative revealed the following improvements in 4-h target performance nationally [3]:

$\begin{array}{lll} & 2002 & \text { September } 2003 \\ \text { Wave 1 } & 83.4 \% & 90 \% \\ \text { Wave 2 } & 72.4 \% & 89.3 \% \\ \text { Wave 3 } & 75.45 \% & 88.7 \% \\ \text { Wave 4 } & 80.39 \% & 91.6 \%\end{array}$

The continuing trends are reflected in data from Barnet and Chase Farm Hospitals NHS Trust in North London. Our hospitals' performance in terms of the 4-h target is detailed below:

The initial target was $90 \%$ of patients should be seen, treated and discharged within $4 \mathrm{~h}$ up to 2004

$\begin{array}{lll} & \text { Performance } & \text { Total attendances } \\ 2002 / 2003 & 71.9 \% & 113,915 \\ 2003 / 2004 & 80.55 \% & 125,269\end{array}$

The target moved to $98 \%$ of patients to be seen, treated and discharged within $4 \mathrm{~h}$ from 2004

\begin{tabular}{lll}
$2004 / 2005$ & $88.5 \%$ & 137,251 \\
$2005 / 2006$ & $95.05 \%$ & 146,758 \\
$2006 / 2007$ & $97.55 \%$ & 148,436 \\
$2007 / 2008$ & $99.1 \%$ & Figures being verified \\
\hline
\end{tabular}

Source: http://www.performance.doh.gov.uk/hospitalactivity/datarequests/ index.htm

\section{Patients with minor injury and illness}

For this group of patients, it was found to be more efficient to stream them into a separate queue and to fast-track them through the ED [4]. The process of triage, wherein their priorities were assessed and they rejoined the queue of patients waiting to be seen, was felt to be counterproductive. Combining triage and rapid clinical assessment maximised the potential from the first contact with a clinician, whether nurse or doctor. Most complaints initiated by patients at the time against EDs related to long waits for attenders with low acuity illness.

Many patients could be seen, treated and discharged home after this first contact - a process referred to as "see and treat". This process required senior clinician involvement, with deployment of these clinicians at the front end of the department. Increasing investment in senior medical and nurse staffing of EDs in the UK allowed for implementation of this change.

For those patients who needed further investigation and treatment in the ED, emergency nurse practitioners, who were able to autonomously assess, investigate, treat and discharge these patients, became important in care delivery. These professionals worked to a pre-determined scope of practice.

\section{Patients requiring further assessment, observation, diagnosis and treatment}

Recognition of groups of patients requiring protocol-driven time-limited assessment led to the proliferation of clinical 
decision units, e.g. for low to moderate risk chest pain. Observation wards attached to the ED, under the administrative charge of emergency medicine specialists, increased in number. These wards admitted a wider range of patients for periods ranging from 6 to $48 \mathrm{~h}$ [5]. The process of counting against the target ceased once the patient was admitted to the ward.

Increasing super-specialisation influenced the appointment of specialists in acute medicine for optimal care of acute medical admissions in the first 24-48 h. These specialists worked in close collaboration with their emergency medicine colleagues and were often present for part of their working time in the ED [6].

Admission avoidance schemes led to development of integrated care pathways for patients with conditions such as suspected deep vein thrombosis and suspected renal colic, allowing for ambulatory investigation and treatment. Community treatment schemes were also developed for conditions such as soft tissue infections including cellulitis and postoperative wound infections.

Fast track, often one-stop, clinics were developed for a range of conditions including transient ischaemic attack, chest pain, recurrent falls, first seizures, haematuria and early pregnancy diagnostic units, among others.

The need for functional assessments of the elderly prior to safe discharge led to the availability of physiotherapists, occupational therapists and social workers within the ED, often working as part of rapid response teams. This facilitated early discharge and effective community care provision.

Some areas of change in practice overlap with those for the subsequent groups under discussion and will be mentioned here. Guided by protocols, nurses could initiate initial investigations, such as blood tests and plain film radiology, in specific patient groups prior to contact with decision-making clinicians. They were also empowered to make direct referrals of certain patients directly to inpatient specialty teams, while the ED staff performed initial assessment and stabilisation. Blood tests, insertion of venous cannulas, urine dipstick testing and electrocardiograms were performed by ED assistants.

\section{Patients requiring acute admission under the medical team}

There was a significant increase in acute medical admissions in the UK, largely related to an ageing population. Clinical algorithms and agreed pathways (e.g. falls) allowed for standardised care. Improved access to intermediate care and step-down care units benefited patients requiring functional rehabilitation. Rapid access to diagnostics, including endoscopy, ultrasound and computed tomography (CT) scanning, allowed for earlier decision making.

\section{Patients requiring acute admission under the surgical team}

Conflicts with elective activity, the effects of superspecialisation, and the proliferation of less experienced junior surgical staff have led to challenges in dealing with acute surgical admissions.

The increased availability of emergency ultrasound and $\mathrm{CT}$ allowed for earlier decision making in the ED. Increased access to day case theatres for emergency surgery improved the responsiveness of the system to emergency admissions. Surgical assessment units with dedicated middle grade surgical support allowed for active observation of patients who might potentially require emergency surgery.

\section{Bed management}

Effective co-ordination of discharges allowed for optimal use of hospital bed capacity. Proactive discharge planning was commenced increasingly at the time of admission, with definition of an anticipated discharge date.

Most hospitals held daily or twice daily performance meetings where current activity was mapped to bed availability and to planned discharges. This allowed for better co-ordination with elective, especially surgical, activity and for redeployment of resources if required to deal with increased emergency activity. At these meetings, all the preceding day's "breaches", i.e. patients waiting $4 \mathrm{~h}$ or longer in the department, were discussed on a case-bycase basis to identify underlying contributory themes.

\section{Escalation policies}

Escalation policies allowed for consistent responses to ED overload. These included pre-defined triggers (increased activity, long waiting times) for activation, usually by site or bed managers, and gradations of response based often on traffic light (red, amber, green) analogies. The roles and responsibilities of key individuals were set out in the form of action cards. A step-up plan allowed for identification of additional bed placements if other activities failed to release sufficient capacity. There is some variation in the effectiveness of these policies, which require effective policing for continued benefit. 
For individual patients, patient management decisions were required to be made by ED staff within $90-120 \mathrm{~min}$ after arrival. If this was not happening, additional senior input was initiated. This activity was often monitored by a nurse acting as a flow co-ordinator.

\section{Pitfalls with implementation of 4-h targets}

Any innovation has potential pitfalls. Target-driven care may distort clinical priorities. Target exemptions have been allowed for patients requiring active ongoing resuscitation when transfer would be detrimental and for patients who unexpectedly deteriorate requiring immediate resuscitation.

Resources dedicated to meeting the target were felt by some not to have improved patient outcomes. However, there is overwhelming evidence of improved patient and provider satisfaction [7], which is an important outcome in its own. Furthermore, the management of time-dependent conditions such as myocardial infarction, major trauma and stroke has benefited from a focus on timely delivery of care.

\section{Conclusions}

The whole systems approach [8] to re-engineering emergency care has led to universal improvements in patient throughput in EDs in the UK. Several of the concepts found to be useful in the NHS are worthy of consideration and adoption by other health care systems. Long waits in the ED are a thing of the past in the UK.

Conflicts of interest None.

\section{References}

1. Improvement in emergency care: case studies. Emergency Services Collaborative, NHS Modernisation Agency, January 2003

2. Ideal Design of Emergency Access (IDEA) Programme, NHS Modernisation Agency National Report, January 2002

3. Emergency Services Collaborative Monthly National Report, September 2003. http://www.modern.nhs.uk/emergency

4. Cooke MW, Wilson S, Pearson S (2002) The effect of a separate stream for minor injuries on accident and emergency department waiting times. Emerg Med J 19:28-30

5. Cooke MW, Higgins J, Kidd P (2003) Use of emergency department observation and assessment wards: a systematic literature review. Emerg Med J 20:138-142

6. Acute medical care. The right person, in the right setting-first time. Acute Medicine Task Force Report. Royal College of Physicians, London, October 2007

7. Mortimore A, Cooper S (2007) The "4-hour target": emergency nurses' views. Emerg Med J 24:402-404

8. Cooke M, Fisher J, Dale J et al (2004) Reducing attendance and waits in emergency departments. A systematic review of present innovations. Report to the National Coordinating Centre for NHS Service Delivery and Organisation R \& D (NCCSDO) 